\title{
The identification of improvement strategies in continuous assessment using sentiment analysis in the Operational Research course
}

\author{
Leonardo H. Talero-Sarmiento ${ }^{a}$, Julián A. Duran-Peña ${ }^{b}$, Kevin A. Salcedo-Rugeles ${ }^{c}$, \\ Sergio M. Garcia-Franco ${ }^{d}$ \\ ${ }^{a}$ M.Sc. Industrial Engineer, Assistant Professor at Universidad Autónoma de Bucaramanga, \\ Bucaramanga, Colombia, e-mail: ltalero@unab.edu.co http://orcid.org/0000-0002-4129-9163, \\ ${ }^{\mathrm{b}} \mathrm{Ph}$.D.(c) Engineer and Industrial Production at Universitat Politècnica de València, Professor at

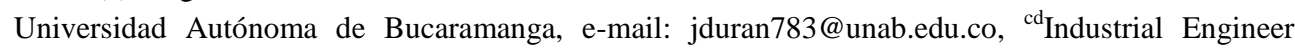 \\ Bachelor student at Universidad Autónoma de Bucaramanga, email: ksalcedo@unab.edu.co and \\ sgarcia720@unab.edu.co.
}

\begin{abstract}
The University aims is to graduate professionals with high levels of competence to impact society positively. In consequence, the institutions apply different educational strategies to focus on improving the curricular competencies until mastery the whole competences topics. An alternative highly applied is continuous assessment, which is a form of educational examination that evaluates the progress of a student throughout a prescribed course. A critical course in the engineer formation is Operational Research; this course focuses on scientific management supported by mathematical models such as decision theory, stochastic scenarios, simulation, mathematical optimization etcetera. The goal of this work is to diagnose the continuous assessment strategy apply to Industrial and System engineer students enrolled in Operational Research course, to do that, applying a sentiment analysis which is a text classification tool that analyses an incoming message (in this case a perception essay) and indicates whether the underlying sentiment is positive, negative or neutral. Furthermore, the Techniques applied to group the emotions of anger, anticipations, disgust, fear, joy, negative, positive, sadness, surprise, and trust. Taking into account the initial results, the authors highlight alternatives such as the flipped classroom, gamification as educational strategies to implement in futures courses looking to improving the continuous assessment positive perception.
\end{abstract}

Keywords: Continuous assessment strategy, Operational Research, Sentiment Analysis. 
The identification of improvement strategies in continuous assessment using sentiment analysis in the operational research course

\section{Introduction}

The bachelor engineers from Universidad Autónoma de Bucaramanga (UAB) are capable of leading industrial processes with social and environmental responsibility. Moreover, the professionals' profiles are diverse such as innovator engineers, directors, public employees, and entrepreneurs, among others. For this, the UAB has been working in three institutional values: 1) Ethics 2) Aesthetic and 3) Logic; this last one cover the different learningteaching strategies which every professor with autonomy could apply in the different Faculties (Universidad Autónoma de Bucaramanga, 2012). Framed in this academic freedom, some professors apply continuous assessment of knowledge and skills as education methodology, because it allows creating a summative assessment, which evaluates the learner at the end of the course (Delgado \& Oliver Cuello, 2006).

Furthermore, as Núñez-Peña et al. cite (2013), this methodology permits to reduce the stress level of the students since, some of them feel panic, helplessness, and disorganization mental when they are required to solve a mathematical problem (Richardson \& Suinn, 1972). Taking into account the continuous assessment benefits, during the first semester of 2019, the authors applied this methodology to the students enrolled in Operational Research course considering productive activities such as software developing tasks, group test, interactive participation, fieldwork, among others. Taking into account preliminaries works is possible to apply machine learning techniques using non-structure data to assess the students' experiences (e.g., academic performance, methodology perceptions, classroom management, and teacher theme domain) (Duarte-Duarte, Talero-Samiento, \& AriasTabares, 2019). In this work, we conduct and quantitative evaluation using sentiment analysis or emotion artificial intelligence, this is one of the fastest-growing research areas in computer science, making it challenging to keep track of all the activities in the area (Mäntylä, Graziotin, \& Kuutila, 2018). Sentiment analysis is a method to systematically identify, extract, quantify, and study affective states and subjective information (Hartman, Stone, Dunphy, Smith, \& Ogilvia, 1967; Liu, 2010) applied to reviews and survey responses.

In consequence, this work has three more phases. In Section 2, are expose the steps to transform the answers into numerical representations and its representative analysis, in Section 3, there are the main results structured by polarity and sentiments. Finally, Section 4 contains the conclusions and discussions of this work. 


\section{Materials and Methods}

\subsection{Data collection}

The authors construct a database from the answers of an open-ended survey focuses on the experiences of 39 students during the Operational Research course. The students are from the Industrial and Systems Engineers programs at UAB courses (4955, 5106 and 52540) located in the city of Bucaramanga, Colombia. The students wrote about their expectation, desires, and improved opportunities with answers length between 400 and 600 words in the Spanish language. As the next step, and to prepare the corresponding analysis, the authors generate 38 different documents in flat texts format and translate verbatim to English for the present manuscript.

\subsection{Data transformation}

This work uses a specific format called tidy tables (an answer transformation structure), where each variable is a column (in this case Group, Gender, and Answer), each observation is a row (a student perception or answer), and each type of observational unit is a table. The tidy text format as being a table with one-token-per-row. A token is a meaningful unit of text, such as a word, that the researchers are interested in using for analysis, and tokenization is the process of splitting text into tokens. This one-token-perrow structure is in contrast to the ways text is often stored in current analyses, perhaps as strings or in a document-term matrix (Fay, 2018). In order to make the transformation, we use the Tidytext package programmed in statistical software $\mathrm{R}$ by Silge \& Robinson (2016), because this package allows the data manipulation with a standard set of "tidy" tools: dplyr (Wickham, Francois, Henry, \& Müller, 2019), tidyr (Wickham \& Henry, 2017), ggplot2 (Wickham, 2009), and broom (Robinson, 2017).

\subsection{Polarity}

The polarity of the perceptions could be calculated after a transformation of the answers from the structure of the tidy table to a word frequency list, for this, the algorithm analyzes which words are used most frequently in the answers, later, the words contained in the list are contrasted to a lexicon dictionary. This phase applies the lexicon created by Bing Liu which includes 2006 positive words, 4783 negative words and includes mis-spellings, morphological variants, slang, and social-media mark-up (Ding, Liu, \& Yu, 2008). After calculating the tokens polarity, those values are added to obtain an overall polarity to each answer. 


\subsection{Sentiment composition}

The perceptions can be cluster by the emotions which they contain. (Kiritchenko, Zhu, \& Mohammad, 2014), to do that, the words in the list are contrasted to the Word-Emotion Association Lexicon, this lexicon is a list of English words and their associations with eight basic emotions (anger, fear, anticipation, trust, surprise, sadness, joy, and disgust) and two sentiments (negative and positive) (Mohammad \& Turney, 2013). After the allocation of the emotions by Group; they are analyzed in percentage format to make comparisons.

\section{Results}

Showing up next the overall sentiments (including emotions). Figure 1, represents the three courses (a.k.a Groups); for the most part, the Groups have a positive trend, which means a good perception of the methodology applied by the Professor. The x-axis indicates the answer number and the $y$-axis the overall polarity. On the other hand, Figure 2 shows the main words and their polarity contributions, in this work, the students usually use words related to excellent satisfaction, and improvement desires, the $\mathrm{x}$-axis indicates the polarity and the $y$-axis is a word list. Figure 3 indicates the word frequency separated by polarity; in this research, the negative words results are related to bad experiences in past courses o anxiety when the students solve mathematical problems.

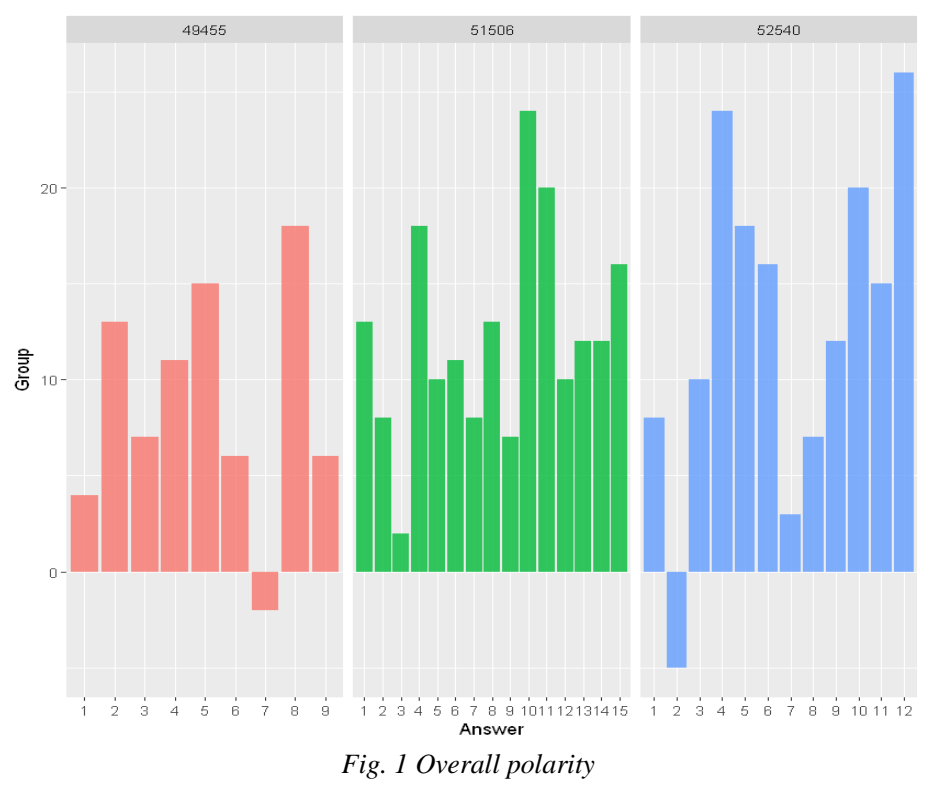

Font: Authors 


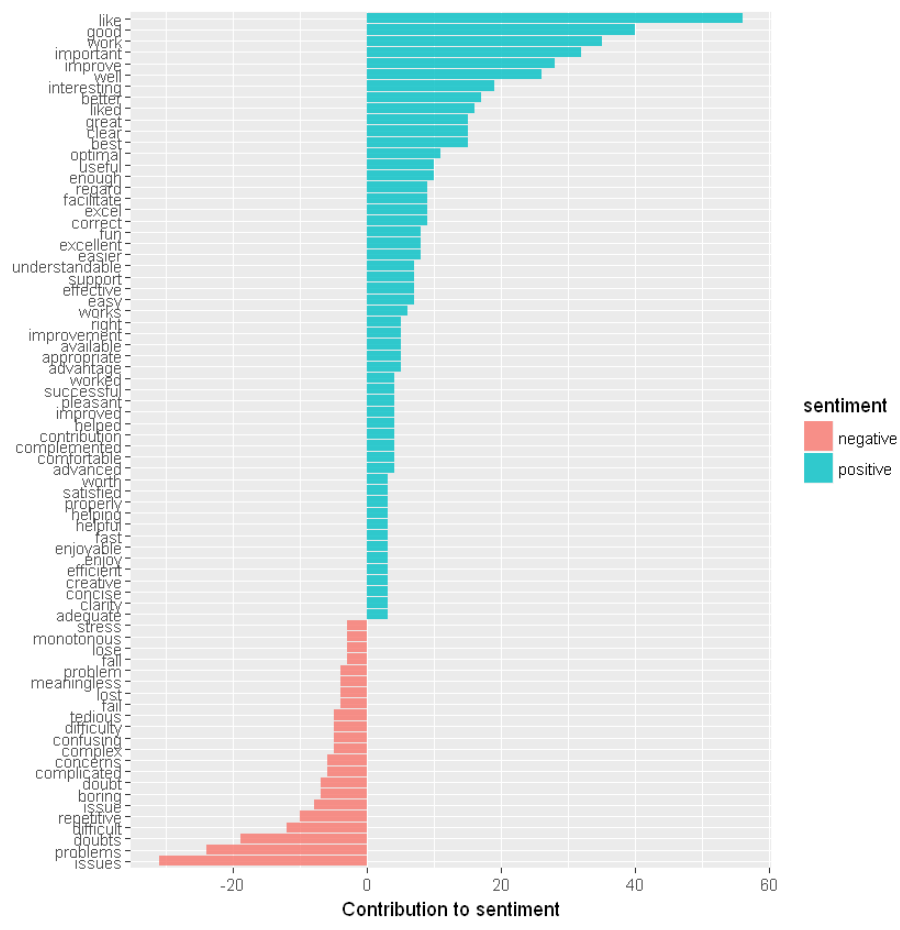

Fig. 2 Contribution to sentiment

Font: Authors

\section{negative}

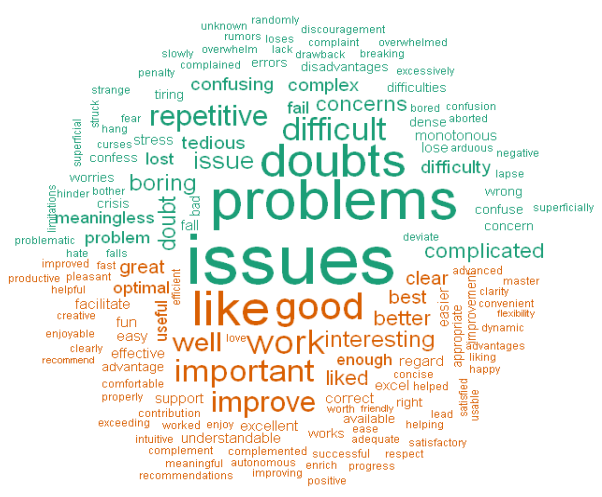

\section{positive}

Fig. 3 World cloud

Font: Authors 
The identification of improvement strategies in continuous assessment using sentiment analysis in the operational research course

Finally, Figure 4 shows the emotions in four clusters, three for each course, and one for the whole students. The percentages indicate that in the 49455 group, the students have the most positive perceptions follow by 51506 group and 52540 . Moreover, 49455 group almost does not have emotions related to adverse perceptions such as fear, sadness, anger, or disgust.

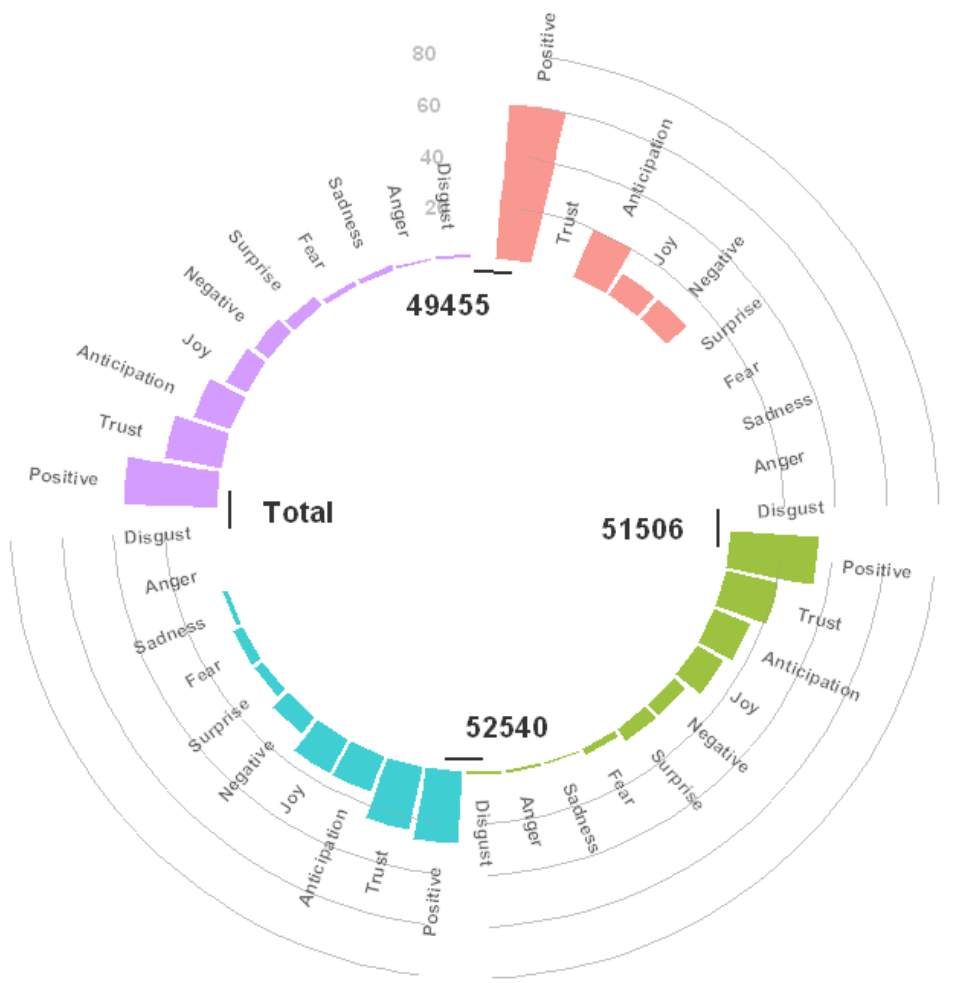

Fig. 4 Sentiments composition by group

Font: Authors

To contextualize the results, we select randomness some students' answers to identity the polarities and emotions. Next, the following quotes are some examples of students' answers. For the most part, the conflicting emotions are related to past experiences in previous courses: "I study this subject for the second time, and it is difficult for me the methodology of the previous teacher, and that is why I lost." Other negative perceptions are related to the anxiety while the students solve mathematical problems: "The subject is complicated for me. So I started a crisis about whether the career I had chosen was the best career for me.". In terms of positive perception, most students feel comfortable with the methodology: "This subject makes me love my profession much more and see how beautiful it is.", "The teacher dominates the subject, and in each class, we entertain in a pleasant 
learning experience. I also want to thank the UAB and the people in charge of the headhunting process.", "I have had the pleasant surprise of receiving more than I expected.", "This subject went from being a boring and uninteresting class to a class that forms levels of logic, analysis; it is also a fun subject.", "I like that the teacher is flexible with the types of work (fieldwork, software development, workshops without a computer) and how he evaluates.". Finally, in terms of opportunities for improvement, students want methodologies such as flipped classroom or gamification: "I would like a platform with extra exercises and explanatory videos to be able to review, learn and resolve doubts." Or "I would like to use several tools oriented to the topics that we see in class and be able to apply them in a company."

\section{Conclusions and discussions}

This research work aims to identify opportunities for improvement in the continuous evaluation strategy to make the student's experience of Operations Research more pleasant in the future since this subject is traditionally stressful and students do not They usually get good grades. For this, we apply the Sentiment Analysis technique since it allows to quantify a polarity and grouping the emotions of the perceptions of the individuals under study. Besides, from applying a filter of emotions to the answers, it was possible to identify strengths, weaknesses, and opportunities for improvement, which makes this work an exciting and fruitful experience. However, the methodology can be improved because in this work the authors translated the answers, and the analysis may be biased, therefore, for future work, it is proposed to work with lexicons in Spanish and make the work more robust by applying machine learning techniques such as recommend Song et al. (2017).

\section{Acknowledgments}

The authors thank all the Operational Research students who participated in the evaluation and the Universidad Autónoma de Bucaramanga.

\section{References}

Delgado, A. M., \& Oliver Cuello, R. (2006). Continuous assessment in the new teaching scenario. RUSC. Universities and Knowledge Society Journal, 3(1). https://doi.org/10.7238/rusc.v3i1.266

Ding, X., Liu, B., \& Yu, P. S. (2008). A holistic lexicon-based approach to opinion mining. In Proceedings of the international conference on Web search and web data mining - 
The identification of improvement strategies in continuous assessment using sentiment analysis in the operational research course

WSDM '08 (p. 231). New York, New York, USA: ACM Press. https://doi.org/10.1145/1341531.1341561

Duarte-Duarte, J. B., Talero-Samiento, L. H., \& Arias-Tabares, J. C. (2019). Identificación de estrategias académicas aplicando minería de texto: El caso de la asignatura Finanzas y Presupuestos. Espacios, 40(21), 6. Retrieved from https://www.revistaespacios.com/a19v40n21/19402106.html

Fay, C. (2018). Text Mining with R: A Tidy Approach. Journal of Statistical Software. https://doi.org/10.18637/jss.v083.b01

Hartman, J. J., Stone, P. J., Dunphy, D. C., Smith, M. S., \& Ogilvia, D. M. (1967). The General Inquirer: A Computer Approach to Content Analysis. American Sociological Review, 32(5), 859. https://doi.org/10.2307/2092070

Kiritchenko, S., Zhu, X., \& Mohammad, S. M. (2014). Sentiment Analysis of Short Informal Texts. Journal of Artificial Intelligence Research, 50, 723-762. https://doi.org/10.1613/jair.4272

Liu, B. (2010). Sentiment Analysis and Subjectivity in: Handbook of Natural Language Processing, Second Edition. Handbook of Natural Language Processing, Second Edition.

Mäntylä, M. V., Graziotin, D., \& Kuutila, M. (2018). The evolution of sentiment analysisA review of research topics, venues, and top cited papers. Computer Science Review, 27, 16-32. https://doi.org/10.1016/j.cosrev.2017.10.002

Mohammad, S. M., \& Turney, P. D. (2013). Crowdsourcing a word-emotion association lexicon. Computational Intelligence, 29(3), 436-465. https://doi.org/10.1111/j.14678640.2012.00460.x

Núñez-Peña, M. I., Suárez-Pellicioni, M., \& Bono, R. (2013). Effects of math anxiety on student success in higher education. International Journal of Educational Research, 58, 36-43. https://doi.org/10.1016/j.ijer.2012.12.004

Richardson, F. C., \& Suinn, R. M. (1972). The Mathematics Anxiety Rating Scale: Psychometric data. Journal of Counseling Psychology, 19(6), 551-554. https://doi.org/10.1037/h0033456

Robinson, D. (2017). broom: Convert Statistical Analysis Objects into Tidy Data Frames. Retrieved from https://cran.r-project.org/package=broom

Silge, J., \& Robinson, D. (2016). tidytext: Text Mining and Analysis Using Tidy Data Principles in R. The Journal of Open Source Software, 1(3), 37. https://doi.org/10.21105/joss.00037 
Song, J., Kim, K. T., Lee, B., Kim, S., \& Youn, H. Y. (2017). A novel classification approach based on Naïve Bayes for Twitter sentiment analysis. KSII Transactions on Internet and Information Systems, 11(6). https://doi.org/10.3837/tiis.2017.06.011

Universidad Autónoma de Bucaramanga. (2012). Proyecto educativo institucional UNAB 2012. Bucaramanga.

Wickham, H. (2009). ggplot2: Elegant Graphics for Data Analysis. Springer-Verlag New York. Media. https://doi.org/10.1007/978-0-387-98141-3

Wickham, H., Francois, R., Henry, L., \& Müller, K. (2019). Package ‘dplyr'. A Grammar of Data Manipulation. $R$ Package Version 0.8.0.1.

Wickham, H., \& Henry, L. (2017). tidyr: Easily Tidy Data with "spread()" and "gather()" Functions. Retrieved from https://cran.r-project.org/package=tidyr 